\title{
Concept of Differential Reflectivity as Applied to the Re- flection of Beam-Limited Radiation by a Convex Body
}

\author{
A. Erteza, J. A. D. Doran, ana H. Lenhert \\ Contribution From the Engineering Experiment Station, University of New Mexico, Albuquerque, \\ N. Mex.
}

(Received April 27, 1964; revised October 9, 1964)

\begin{abstract}
A method is presented for obtaining the backscattered radiation intensity from an idealized target illuminated by a beamwidth limited source using the concept of differential reflectivity. In general, this concept provides a method for determining the fields reflected from a body illuminated by an arbitrary source or antenna pattern when the reflection coefficients for a plane wave incident on the body are known or approximated. Fresnel's reflection coefficients are claimed to be appropriate approximations for the class of convex targets characterized by either a large radius of curvature or consisting of lossy material. In the application of this method to the case of a large spherical body, considerable computational simplification results. Application of results to appropriate experimental data yields a minimum value of 1.53 for the average dielectric constant of the moon's surface material. Extension of the method to statistical problems is indicated.
\end{abstract}

\section{Introduction}

In recent years numerous attempts have been made to obtain information on the properties of such distant bodies as the moon and neighboring planets [Beckmann, 1963]. A major method involves analyzing and interpreting the backscattered radar return from these bodies. This backscattered radar return may be analyzed from a number of different aspects, depending on the nature of information sought. Through statistical analysis, such information as pulse ensemble averages for discrete times during the return as well as autocorrelations, cross correlations, and variances thereof may be found. But even for a body as close as the moon, the information that can be obtained from such analyses will represent gross averages over large areas of the body.

Information of particular interest is the value of each of the electromagnetic properties, such as $\epsilon, \mu$, and $\sigma$, which even in simple nonstatistical problems are extremely difficult to separate from each other. However, this separation can be achieved when the data is examined in the light of some simplifying theory capable of predicting the return for sharply defined ideal conditions. An analysis of the return data together with the theoretical solution should then yield average values for the electromagnetic quantities sought.

This paper presents a solution describing the radar return from a simple geometric shape and an application of this solution to the case of an idealized moon. In the case of the moon, the idealization is necessary to de-emphasize the contribution of factors such as roughness and local variations in electromagnetic properties. The derivation of the integral representing the radar return is rigorous, but certain simplifying assumptions are necessary to carry out the integration in closed form.

${ }^{1}$ This work sponsored by the National Aeronautics and Space Administration under Grant NsG 1 \&9-61. 


\section{Theory}

\subsection{Concept of Differential Reflectivity}

A concept which will prove useful in the development of the theory outlined in this paper is that of "differential reflectivity." This is a dyadic quantity. When this dyadic is multiplied by a differential surface area and the vector field incident on that area, there results an expression of the contribution of that surface element to the scattered field at an arbitrary observation point. The differential reflectivity is a function of the following:

(1) Location and orientation of the surface with respect to the observation point.

(2) Properties of the two media separated by the surface.

(3) Frequency of the incident radiation, $\omega$.

For the case of steady state incident radiation the reflected Hertzian potential field, for instance, may be described by

$$
\vec{\Pi}_{r}\left(\vec{r}_{1}, \omega, t\right)=\iint_{S_{0}} \hat{\sigma}\left(\vec{r}_{1}, \vec{r}_{0}, \omega\right) \cdot \vec{\Pi}_{i}\left(\vec{r}_{0}, \omega, t\right) d S_{0}
$$

where $\vec{r}_{1}$ and $\vec{r}_{0}$ denote the coordinates of the field point and the surface points, respectively; $\vec{\Pi}_{i}\left(\vec{r}_{0}, \omega, t\right)$ is the incident vector field at the surface $S_{0}$; and $\left.\hat{\sigma} \overrightarrow{r_{1}}, \overrightarrow{r_{0}}, \omega\right)$ is the dyadic differential reflectivity.

Consider now that the radiation incident on a surface is from a pulsed, "conical" source. A "conical" source is understood to be one whose radiation is limited to a cone of vertex angle $\alpha$. Within the cone the electric and magnetic fields are uniform over any spherical surface centered on the source. Exterior to the cone they are identically zero. Let the source radiate between times $t=0$ and $t=T$ a sinusoidally varying carrier wave of angular frequency $\omega_{0}$. At some later time the outwardly traveling wave impinges on an infinite plane surface $(z=0$ in fig. 1) which separates all of space into two semi-infinite regions, each of which is filled with a homogeneous medium. The portion of the plane intercepted by the cone is given by $S_{0}$. The intensity of the backscattered field at an arbitrary observation point is desired.

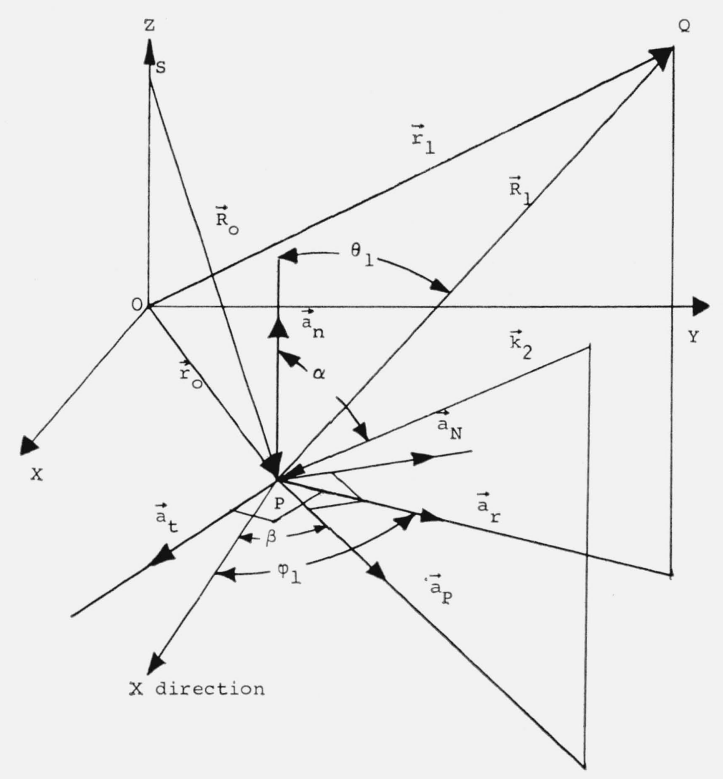

Figure 1. Reflection geometry. 
If one replaces the incident wave packet with an infinite set of steady state (conically bounded) incident waves obtained by means of the Fourier transform, one may solve for the contribution of a typical member of the family of steady state waves and, finally, sum up or integrate these contributions over the whole family to obtain the solution to the original problem. It may be observed that Weyl's method of expansion into plane waves [Stratton, 1941, pp. 577-582] is not applicable to the beamwidth limited case.

Using the expression for the differential reflectivity, the contribution to the total field at the observation point due to a conical bounded typical member of the family of steady state waves can be written as

$$
\left.\left.\Delta \vec{\Pi}_{r} \vec{r}_{1}, \omega_{0}, \omega\right)=\iint_{S_{0}} \hat{\sigma}\left(\vec{r}_{1}, \vec{r}_{0}, \omega\right) \cdot \vec{\Pi}_{i} \vec{r}_{0}, \omega_{0}, \omega\right) d S_{0}
$$

where $\hat{\sigma}\left(\vec{r}_{1}, \vec{r}_{0}, \omega\right)$ is the differential reflectivity and $\overrightarrow{\mathrm{II}}_{i}\left(\vec{r}_{0}, \omega_{0}, \omega\right)$ is the complex phasor for the incident field due to the steady state wave of angular frequency $\omega$. The time dependent total scattered field is then

$$
\left.\vec{\Pi}_{r}\left(\vec{r}_{1}, \omega_{n}, t\right)=\frac{1}{2 \pi} \int_{-\infty}^{\infty} e^{-i \omega t}\left[\int_{S_{0}}^{\infty} \hat{\sigma}\left(\vec{r}_{1}, \vec{r}_{0}, \omega\right) \cdot \vec{\Pi}_{i} \vec{r}_{0}, \omega_{0}, \omega\right) d S_{0}\right] d \omega .
$$

\subsection{Theory of Differential Reflectivity}

With attention focused on only one member of the family of steady state waves, a derivation of the theory involving the concept of differential reflectivity will now be shown. Let the Hertz vector due to the component steady state incident wave be described by

$$
\vec{\Pi}_{i}\left(\vec{r}_{\mathrm{c}} . \omega, t\right)=\vec{a}_{\pi} C_{0} \frac{e^{i k_{2} R_{0}}}{R_{0}} e^{-i \omega t}
$$

for all points $\vec{r}_{0}$ on the surface $S_{0}$. On the remainder of the infinite plane surface it is identically zero. Here $\vec{a}_{\pi}$ is the unit vector in the $\vec{\Pi}_{i}$ direction, $C_{0}=C_{0}(\omega)$ and relates to the source strength, $k_{2}=k_{2}(\omega)$ is the propagation constant in the incident medium, and $\overrightarrow{R_{0}}=\overrightarrow{r_{0}}-\overrightarrow{r_{s}}$, where $\rightarrow$ $r_{s}$ is the radius vector from the origin to the source point.

If the origin is taken in the infinite plane surface (the $x-y$ plane) of which $S_{0}$ is a region, and the source has the rectangular coordinates $\left(0,0, z_{s}\right)$ as shown in figure 1 , so that

there can be written

$$
R_{0}=\left|\vec{r}_{0}-\vec{r}_{s}\right|=\sqrt{x_{0}^{2}+\zeta_{0}^{2}+z_{s}^{2}}
$$

$$
\vec{\Pi}_{i}\left(\vec{r}_{0}, \omega, t\right)=\vec{a}_{\pi} C_{0} e^{-i \omega t} \iint_{S_{0}} \frac{e^{i k_{2} R_{0}^{\prime}}}{R_{0}^{\prime}} \delta\left(x_{0}-x_{0}^{\prime}\right) \delta\left(y_{0}-y_{0}^{\prime}\right) d x_{0}^{\prime} d y_{0}^{\prime}
$$

where

$$
R_{0}^{\prime}=\sqrt{x_{0}^{\prime 2}+y_{0}^{\prime 2}+z_{s}^{2}}
$$

Using the Fourier integral expansion one may write

$$
\delta\left(x_{0}-x_{0}^{\prime}\right) \delta\left(y_{0}-y_{0}^{\prime}\right)=\frac{1}{4 \pi^{2}} \int_{-\infty}^{\infty} \int^{i u\left(x_{0}-\lambda_{0}^{\prime}\right)+i v\left(y_{6}-y_{0}^{\prime}\right)} d u d v
$$

so that the incident field at each point, $\vec{r}_{0}$, of the surface is given by

$$
\vec{\Pi}_{i}\left(\vec{r}_{0}, \omega, t\right)=\frac{e^{-i \omega t}}{4 \pi^{2}} \iint_{S_{0}} \frac{e^{i k_{2} R_{0}^{\prime}}}{R_{0}^{\prime}}\left[\iint_{-\infty}^{\infty} \vec{a}_{\pi} C_{0} e^{i u\left(x_{0}-x_{0}^{\prime}\right)+i v\left(z_{0}-y_{0}^{\prime}\right)} d u d v\right] d x_{0}^{\prime} d y_{0}^{\prime} .
$$


If now $r=\sqrt{x_{0}^{2}+y_{0}^{2}+z_{0}^{2}}$ there can be obtained by analytic continuation the expression

$$
\vec{\Pi}_{i}(\vec{r}, \omega, t)=\frac{e^{-i \omega t}}{4 \pi^{2}} \iint_{S_{0}} \frac{e^{i k_{2} R_{0}^{\prime}}}{R_{0}^{\prime}}\left[\int_{-\infty}^{\infty} \int_{a_{\pi}} \vec{a}_{0} C_{0} e^{i W_{i}} d u d v\right] d x_{0}^{\prime} d y_{0}^{\prime}
$$

where

$$
W_{i}=u\left(x_{0}-x_{0}^{\prime}\right)+v\left(y_{0}-y_{0}^{\prime}\right)-z_{0} \sqrt{k_{2}^{2}-\left(u^{2}+v^{2}\right)} .
$$

The expression (8) which reduces to $(7)$ for $\vec{r}=\vec{r}_{0}$ is subject to the following interpretation: it can be considered to be the field due to an infinite collection of plane waves, symmetrically distributed about the local normal to the incident wave front, which combine at a point on the reflecting surface to yield the net incident field due to the original source. The propagation constant associated with each of the plane waves is so determined that the entire collection adds to a two-dimensional delta function at the point in question.

Consider now a Hertzian plane wave having a propagation vector with components $\left[u, v, \sqrt{k_{2}^{2}-\left(u^{2}+v^{2}\right)}\right]$ and polarization in the direction $\vec{a}_{\pi}$ to be reflected from the surface. The reflected plane wave will be described through the use of a dyadic reflection coefficient $\hat{V}(u, v)$. At an observation point $Q\left(\overleftarrow{r_{1}}\right)$ the total reflected field due to illumination of the surface $S_{0}$ by the infinite set of plane waves will be given by

$$
\vec{\Pi}_{r}\left(\vec{r}_{1}, \omega, t\right)=\iint_{S_{0}}\left(\left[\frac{1}{4 \pi^{2}} \int_{-\infty}^{\infty} \hat{V}(u, v) e^{i w_{r}} d u d v\right] \cdot\left[\vec{a}_{\pi} C_{0} \frac{e^{i k_{2} R_{0}^{\prime}}}{R_{0}^{\prime}} e^{-i \omega t}\right]\right) d x_{0}^{\prime} d y_{0}^{\prime}
$$

where

$$
W_{r}=u\left(x_{1}-x_{0}^{\prime}\right)+v\left(y_{1}-y_{0}^{\prime}\right)+z_{1} \sqrt{k_{2}^{2}-\left(u^{2}+v^{2}\right)} .
$$

Comparison with (1) yields the differential reflectivity

$$
\hat{\sigma}=\frac{1}{4 \pi^{2}} \int_{-\infty}^{\infty} \hat{V}(u, v) e^{i W_{r}} d u d v .
$$

The form of the components of the reflection coefficient $\hat{V}$ will depend on the nature of the surface $S_{0}$. If the surface is spherical they may be derived from Mie's solution for a plane wave incident on a sphere [Stratton, 1941, pp. 563-567]. For an infinite plane surface they reduce to the ordinary Fresnel reflection coefficients.

\subsection{Derivation of the Components of $\hat{\sigma}$}

For purposes of computation it will prove useful to evaluate the vector quantity

$$
\vec{\Sigma}=\frac{1}{4 \pi^{2}} \int_{-\infty}^{\infty} \int_{\hat{V}} \hat{V}(u, v) \cdot \overrightarrow{a_{\pi}} C_{0} e^{i W_{r}} d u d v=\hat{\sigma} \cdot \overrightarrow{a_{\pi}} C_{0} .
$$

Referring to figure 1, let there be defined two coordinate systems having their origins at the point $P$ on the reflecting surface. The $Q$ system will be defined by the orthogonal set of unit vectors $\vec{a}_{t}, \vec{a}_{r}$ and $\vec{a}_{n}$ and the $K$ system by the orthogonal set $\vec{a}_{P}, \vec{a}_{N}$ and $\vec{a}_{n}$. Here $\vec{a}_{n}$ is the positive unit normal to the reflecting surface, $\vec{a}_{r}$ is in the direction of the projection of $\vec{R}_{1}$ on the tangent plane through $P$, and $\overrightarrow{a_{t}}=\vec{a}_{r} \times \vec{a}_{n}$. The vector $\vec{a}_{P}$ is in the direction opposite to that of the projection of $\vec{k}_{2}$ on the tangent plane and $\overrightarrow{a_{N}}=\overrightarrow{a_{n}} \times \overrightarrow{a_{P}}$.

These two reference systems are in addition to the primary reference system in which, in a generalized problem, the surface normal changes direction as one traverses the surface under consideration. 
The vector (11) may be resolved as follows:

$$
\begin{aligned}
\vec{\Sigma}=\frac{1}{4 \pi^{2}} \int_{-\infty}^{\infty} \int_{\infty} \vec{a}_{P} V_{P P} C_{P} e^{i W_{r}} d u d v+\frac{1}{4 \pi^{2}} \int_{-\infty}^{\infty} \int_{a_{N}} \vec{a}_{N N} V_{N} e^{i W_{r}} d u d v & \\
& +\frac{1}{4 \pi^{2}} \iint_{-\infty}^{\infty} \vec{a}_{n} V_{n n} C_{n} e^{i W_{r}} d u d v=\sum_{P}+\sum_{N}+\sum_{n}
\end{aligned}
$$

where $\vec{a}_{\pi} C_{0}=\vec{a}_{P} C_{P}+\vec{a}_{N} C_{N}+\vec{a}_{n} C_{n}$. For plane I-waves reflecting from an infinite plane surface it can be shown that

$$
\begin{aligned}
& V_{P P}(\alpha)=-V_{n n}(\alpha)=-\frac{\left(\frac{\mu_{2}}{\mu_{1}}\right) n^{2} \cos \alpha-\sqrt{n^{2}-\sin ^{2} \alpha}}{\left(\frac{\mu_{2}}{\mu_{1}}\right) n^{2} \cos \alpha+\sqrt{n^{2}-\sin ^{2} \alpha}} \\
& V_{N N}(\alpha)=\frac{\left(\frac{\mu_{1}}{\mu_{2}}\right) \cos \alpha-\sqrt{n^{2}-\sin ^{2} \alpha}}{\left(\frac{\mu_{1}}{\mu_{2}}\right) \cos \alpha+\sqrt{n^{2}-\sin ^{2} \alpha}}
\end{aligned}
$$

where $\alpha=$ angle of incidence $=\sin ^{-1}\left(\frac{\sqrt{u^{2}+v^{2}}}{k_{2}^{2}}\right)$, and $n=$ index of refraction $=\frac{k_{1}}{k_{2}}$. In this case $V_{P P}$ and $V_{N N}$ are identical with the Fresnel reflection coefficients. Off-diagonal components of $\hat{V}$ are identically zero by virtue of the choice of reference system.

Let

$$
\begin{aligned}
& u=\lambda \cos \beta=k_{2} \sin \alpha \cos \beta \\
& v=\lambda \sin \beta=k_{2} \sin \alpha \sin \beta \\
& x_{1}-x_{0}^{\prime}=\rho_{1} \cos \varphi_{1} \\
& y_{1}-y_{0}^{\prime}=\rho_{1} \sin \varphi_{1} \\
& \rho_{1}=R_{1} \sin \theta_{1} \\
& z_{1}=R_{1} \cos \theta_{1} .
\end{aligned}
$$

On substitution into (12) one obtains

$$
\begin{aligned}
\sum_{n} & =\vec{a}_{n} \frac{C_{n}}{4 \pi^{2}} \int_{0}^{\infty} V_{n n}[\alpha(\lambda)]\left[\int_{0}^{2 \pi} e^{i \lambda \rho_{1} \cos \left(\varphi_{1}-\beta\right)+i z_{1} k_{2} \cos \alpha} d \beta\right] \lambda d \lambda \\
& =\vec{a}_{n} \frac{C_{n}}{2 \pi} \int_{0}^{\infty} V_{n n}[\alpha(\lambda)] \cdot J_{0}\left(\lambda \rho_{1}\right) e^{i z k_{2} \cos \alpha} \lambda d \lambda .
\end{aligned}
$$

Also one can obtain

$$
\begin{aligned}
\sum_{T}= & \sum_{P}+\vec{\sum}_{N} \\
= & \vec{a}_{t} \frac{C_{t}}{4 \pi} \int_{0}^{\infty}\left[f_{1}(\lambda) J_{0}\left(\lambda \rho_{1}\right)+f_{2}(\lambda) J_{2}\left(\lambda \rho_{1}\right)\right] e^{i z_{1}^{*} k_{2} \cos \alpha} \lambda d \lambda \\
& +\vec{a}_{r} \frac{C_{r}}{4 \pi} \int_{0}^{\infty}\left[f_{1}(\lambda) J_{\theta}\left(\lambda \rho_{1}\right)-f_{2}(\lambda) J_{2}\left(\lambda \rho_{1}\right)\right] e^{i z_{1} k_{2} \cos \alpha} \lambda d \lambda
\end{aligned}
$$

where

$$
\begin{aligned}
& f_{1}(\lambda)=V_{P P}[\alpha(\lambda)]+V_{N N}[\alpha(\lambda)] \\
& f_{2}(\lambda)=V_{P P}[\alpha(\lambda)]-V_{N N}[\alpha(\lambda)]
\end{aligned}
$$




$$
\begin{aligned}
& C_{t}=\left(\vec{a}_{t} \cdot \vec{a}_{\pi}\right) C_{0}=\left(\vec{a}_{t} \cdot \vec{a}_{P}\right) C_{P}+\left(\overrightarrow{a_{t}} \cdot \overrightarrow{a_{N}}\right) C_{N} \\
& C_{r}=\left(\vec{a}_{r} \cdot \vec{a}_{\pi}\right) C_{0}=\left(\vec{a}_{r} \cdot \vec{a}_{P}\right) C_{P}+\left(\vec{a}_{r} \cdot \vec{a}_{N}\right) C_{N} .
\end{aligned}
$$

Approximate evaluation of $\vec{\Sigma}_{n}$ and $\vec{\Sigma}_{T}$ as given in (15) and (16) may be made by use of the saddle-point method [Brekhovskikh, 1960] which, under the assumption $k_{2} R_{1} \sin ^{2} \theta_{1}>>1$, yields

$$
\begin{aligned}
& \vec{\sum}_{n}=-\vec{a}_{n} \frac{k_{2}}{2 \pi i} V_{P P}\left(\theta_{1}\right) \frac{e^{i k_{2} R_{1}}}{R_{1}} \cos \theta_{1} C_{n} \\
& \sum_{T}=\vec{a}_{t} \frac{k_{2}}{2 \pi i} V_{N N}\left(\theta_{1}\right) \frac{e^{i k_{2} R_{1}}}{R_{1}} \cos \theta_{1} C_{t}+\vec{a}_{r} \frac{k_{2}}{2 \pi i} V_{P P}\left(\theta_{1}\right) \frac{e^{i k_{2} R_{1}}}{R_{1}} \cos \theta_{1} C_{r}
\end{aligned}
$$

From the expression $\vec{\Sigma}=\hat{\sigma} \cdot \vec{a}_{\pi} C_{0}$ we now find

$$
\begin{aligned}
\sigma_{i j} & =0 \quad i \neq j \\
\sigma_{t t} & =\frac{k_{2}}{2 \pi i} V_{N N}\left(\theta_{1}\right) \frac{e^{i k_{2} R_{1}}}{R_{1}} \cos \theta_{1} \\
\sigma_{r r} & =\frac{k_{2}}{2 \pi i} V_{P P}\left(\theta_{1}\right) \frac{e^{i k_{2} R_{1}}}{R_{1}} \cos \theta_{1} \\
\sigma_{n n} & =-\frac{k_{2}}{2 \pi i} V_{P P}\left(\theta_{1}\right) \frac{e^{i k_{2} R_{1}}}{R_{1}} \cos \theta_{1}
\end{aligned}
$$

as the components of the differential reflectivity with respect to the designated coordinate system.

Similar results may be obtained for surfaces of arbitrary curvature by use of the appropriate form of the reflection coefficient. It is realized that evaluation of the reflection coefficient $\hat{V}$ for a plane wave incident on an arbitrary surface may be a problem in itself.

\subsection{Discussion on $\hat{\sigma}$}

The differential cross section derived above is readily applicable to the problem of scattering from an infinite plane surface. It is noted from the form of the vector $\vec{\Sigma}$ that the field strength at right angles to the surface normal is zero due to the $\cos \theta_{1}$ factor. In line with our original assumptions then, a plane surface can be considered as a collection (infinite) of independent secondary sources without mutual coupling. This is a remarkable result inasmuch as seldom in 3-dimensional electromagnetic scattering problems can neighbor-to-neighbor interactions be ignored. If, now, a portion of the surface illuminated has the cross-sectional profile shown in figure 2, it is apparent that there will be a contribution to the fields at both $\mathrm{B}$ and $\mathrm{C}$ due to the secondary source at $\mathrm{A}$ and vice versa. The strength of the secondary sources everywhere on the surface will thus be affected. The possibility of secondary reflections such as occur at $\mathrm{B}$ may be negated by requiring that the bodies illuminated be convex.

The situation at $\mathrm{C}$ cannot be so simply disposed of. However, it can be seen that if the radius of curvature of the surface is large, the contribution at $\mathrm{C}$ due to the source at $\mathrm{A}$ will be small. In addition, if the material composing the target body is lossy, the attenuation suffered in traversing the path $\mathrm{A}$ to $\mathrm{C}$ will be so high that the re-radiation from the $\mathrm{C}$ surface element due to the illumination of the A surface element will be negligible. The method outlined herein is, therefore, an excellent approximation for the solution of scattering problems involving large convex bodies or any highly lossy convex body. 

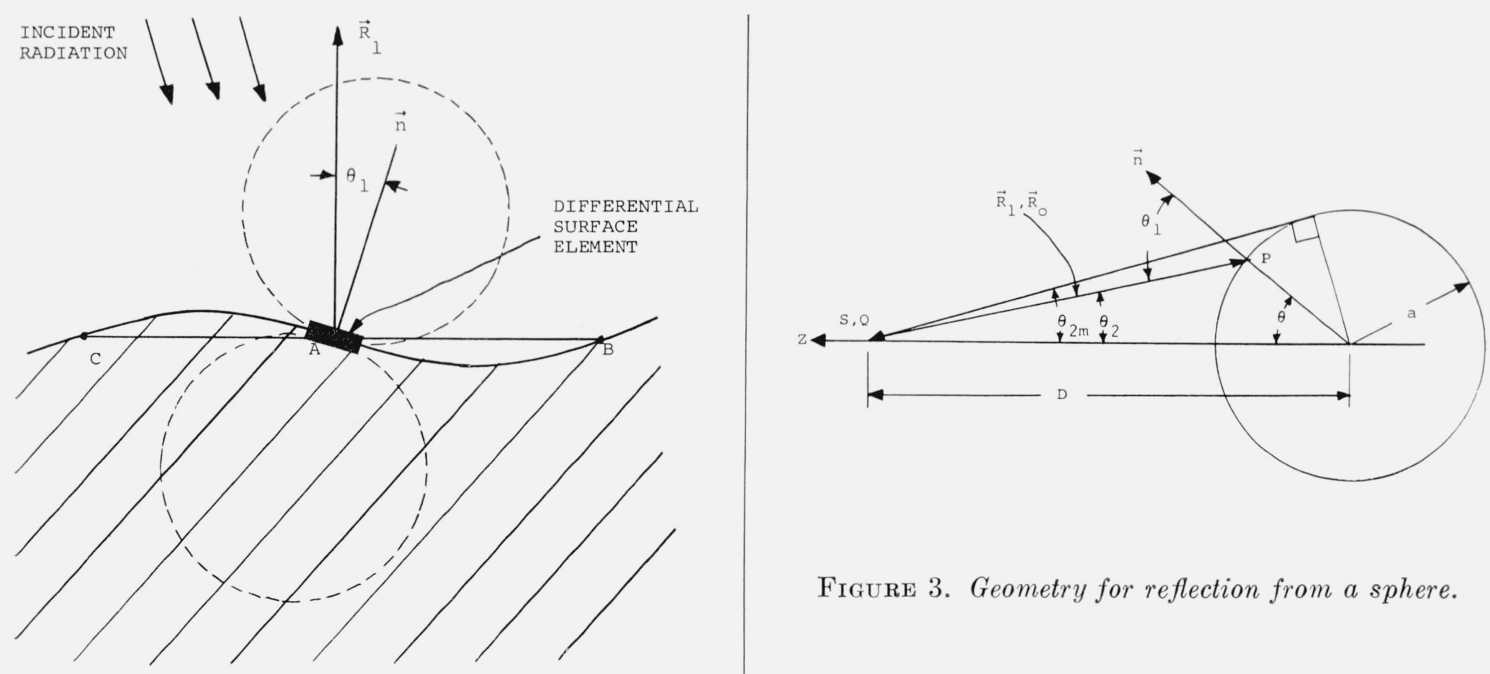

FiguRE 3. Geometry for reflection from a sphere.

Figure 2. Reflection from nonplanar surface.

\section{Application to the Idealized Moon}

An application of the method to the case of a large spherical object such as the moon can easily be made with the aid of a few approximating assumptions. In particular, the spherical object will be assumed to be a smooth sphere of large radius, $a$, and composed of a homogeneous lossless substance. Geometry pertinent to the problem is shown in figure 3. The origin of the spherical coordinate system is located at the center of the sphere with the source and the receiver being located at $r=D$ and $\theta=0$. Additionally, let $\theta=\frac{\pi}{2}$ and $\varphi=0$ define the positive $x$-axis; and $\theta=0$ define the positive $z$-axis.

Let the source be an electric dipole oriented in the $x$-direction so that

$$
\vec{\Pi}_{i}=:=\vec{a}_{x} C_{0} \frac{e^{i k_{2} R_{0}}}{R_{0}}
$$

where $R_{0}$ is the distance from the source to a point on the surface of the sphere. It is readily seen that

$$
\begin{aligned}
& C_{t}=-C_{0} \sin \varphi \\
& C_{r}=-C_{0} \cos \varphi \cos \theta \\
& C_{n}=C_{0} \cos \varphi \sin \theta
\end{aligned}
$$

Realizing that the proper reflection coefficients to use in this case would be those obtained from Mie's solution to the problem of a plane wave incident on a sphere, the assumption is now made that due to the very large radius of the sphere compared to the wavelength of the incident radiation, these reflection coefficients may be replaced by their limiting values as the radius of the sphere increases without limit. These limiting values are, ipso facto, the Fresnel reflection coefficients as given in (13).

From (1), (18), and (20) there is obtained

$$
\begin{aligned}
\vec{\Pi}_{r}=-C_{0} \frac{a^{2} k_{2}}{2 \pi i} \iint_{S} \cos \theta_{1}\left[\vec{a}_{t} \sin \varphi V_{N N}\left(\theta_{1}\right)+\vec{a}_{r} \cos \varphi\right. & \cos \theta V_{P P}\left(\theta_{1}\right) \\
& \left.+\vec{a}_{n} \cos \varphi \sin \theta V_{P P}\left(\theta_{1}\right)\right] \frac{e^{i k_{2} R_{0}}}{R_{0}} \frac{e^{i k_{2} R_{1}}}{R_{1}} \sin \theta d \theta d \varphi
\end{aligned}
$$


where $R_{1}$ is as before, the distance from a point on the surface to the observation point. If in this expression one replaces $R_{1}$ by $R_{0}$ so as to obtain the backscattered field at the position of the source one finds, on performing the integration over $\varphi$, that (21) vanishes. However, as will be shown, the $\vec{E}$ and $\vec{H}$ fields at this point do not vanish. We will then be able to compute the net power incident on an antenna of given effective area.

The elementary far field contributions to the $\vec{E}$ and $\vec{H}$ fields at the observation point are given by

$$
\begin{aligned}
& \delta \vec{E}\left(R_{1}\right)=-k_{2}^{2} \vec{a}_{R} \times\left(\vec{a}_{R} \times \delta \vec{\Pi}_{r}\right) \\
& \delta \vec{H}\left(R_{1}\right)=\omega k_{2} \epsilon_{2}\left(\vec{a}_{R} \times \delta \vec{\Pi}_{r}\right)
\end{aligned}
$$

where

$$
\overrightarrow{a_{R}}=\vec{a}_{r} \sin \theta_{1}+\vec{a}_{n} \cos \theta_{1}
$$

and

$\delta \vec{\Pi}_{r}=-C_{0} \frac{a^{2} k_{2}}{2 \pi i} \cos \theta_{1}\left[\vec{a}_{t} \sin \varphi V_{N N}\left(\theta_{1}\right)+\vec{a}_{r} \cos \varphi \cos \theta V_{P P}\left(\theta_{1}\right)\right.$

$$
\left.+\vec{a}_{n} \cos \varphi \sin \theta V_{P P}\left(\theta_{1}\right)\right] \frac{e^{i k_{2} R_{1}}}{R_{1}} \frac{e^{i k_{2} R_{0}}}{R_{0}} \sin \theta d \theta d \varphi
$$

One obtains, on making the indicated substitutions and integrating over $\varphi$, the remaining components of $\vec{E}$ and $\vec{H}$

$$
E_{x}\left(R_{0}\right)=\frac{\pi a^{2} C_{0} k_{2}^{3}}{2 \pi i} \int_{0}^{\theta_{a}} \frac{e^{i 2 k_{2} R_{0}\left(\theta_{1}\right)}}{D^{2}} \sin \theta_{1} \cos \theta_{1}\left[V_{P P}\left(\theta_{1}\right) \cos 2 \theta_{1}+V_{N N}\left(\theta_{1}\right)\right] d \theta_{1}
$$

and

$$
H_{y}\left(R_{0}\right)=\frac{\pi a^{2} \omega k_{2}^{2} \epsilon_{2} C_{0}}{2 \pi i} \int_{0}^{\theta_{a}} \frac{e^{i 2 k_{2} R_{0}\left(\theta_{1}\right)}}{D^{2}} \sin \theta_{1} \cos \theta_{1}\left[V_{P P}\left(\theta_{1}\right) \cos 2 \theta_{1}+V_{N N}\left(\theta_{1}\right)\right] d \theta_{1}
$$

where $R_{0} \approx D$ and $\theta_{1} \approx \theta$ have been used as approximations since $D>>a$.

In order to obtain numerical results for the problem at hand, the Fresnel reflection coefficients $V_{P P}$ and $V_{N N}$ are replaced at this point by the expressions

$$
\begin{aligned}
& V_{P P}^{\prime}=L_{3} \cos ^{2} \theta+L_{4} \cos \theta+1 \\
& V_{N N}^{\prime}=L_{1} \cos ^{2} \theta+L_{2} \cos \theta-1
\end{aligned}
$$

where

$$
\begin{aligned}
L_{1} & =-0.7 \\
L_{2} & =\rho+1.7 \\
L_{3} & =\frac{\rho}{1-h}+\frac{1}{h} \\
L_{4} & =-\left[L_{3} h+\frac{1}{h}\right] \\
\rho & =V_{N N}(0)=\frac{1-n}{1+n} \text { if } \mu_{1}=\mu_{2}
\end{aligned}
$$

and

$$
h=\cos \theta_{b}
$$


Figure 4. Comparison of exact and approximate reflection coefficients.

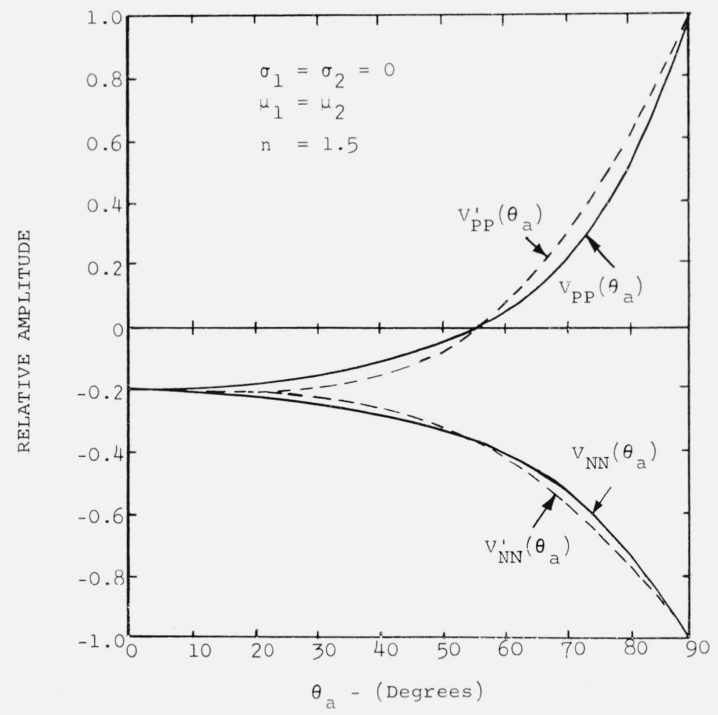

where $\theta_{b}$ is that angle satisfying the relation $V_{P P}\left(\theta_{b}\right)=0$. The approximation is based on matching the curves $V_{P P}(\theta)$ versus $\theta$ and $V_{N N}(\theta)$ versus $\theta$ at three points, namely, at $\theta=0^{\circ}, \theta=90^{\circ}$, and $\theta=\theta_{b}$. Figure 4 shows the approximate reflection coefficients $V_{P P}^{\prime}$ and $V_{N N}^{\prime}$ in relation to the exact coefficients for the case:

$$
\mu_{1}=\mu_{2}, \sigma_{1}=\sigma_{2}=0 \text { and } n=1.5 \text {. }
$$

Let now

$$
\begin{aligned}
& E_{x} \approx \frac{a^{2} C_{0} k_{2}^{3}}{2 D^{2}} I \\
& H_{y} \approx \frac{a^{2} \omega k_{2}^{2} \epsilon_{2} C_{0}}{2 D^{2}} I
\end{aligned}
$$

where

$$
I=-i \int_{0}^{\theta_{a}} e^{i 2 k_{2}\left(D-a \cos \theta_{1}\right)} \sin \theta_{1} \cos \theta_{1}\left[V_{P P}^{\prime}\left(\theta_{1}\right) \cos 2 \theta_{1}+V_{N N}^{\prime}\left(\theta_{1}\right)\right] d \theta_{1}
$$

On substitution of the expressions for $V_{P P}^{\prime}$ and $V_{N N}^{\prime}$ there results, after integration and dropping of terms involving powers of $\frac{1}{2 k_{2} a}$ higher than the first,

$$
I=-\frac{e^{i 2 k_{2} D}}{2 k_{2} a}\left[e^{-i 2 k_{2} a \cos \theta_{a}} f\left(\theta_{a}\right)-f_{0} e^{-i 2 k_{2} a}\right]
$$

where $f_{0}=2 \rho$ and

$$
f\left(\theta_{a}\right)=2 L_{3} \cos ^{5} \theta_{a}+2 L_{4} \cos ^{4} \theta_{a}+\left(L_{1}-L_{3}+2\right) \cos ^{3} \theta_{a}+\left(L_{2}-L_{4}\right) \cos ^{2} \theta_{a}-2 \cos \theta_{a} .
$$

From the relation

$$
\bar{S}=\frac{1}{2} \operatorname{Re}\left(\vec{E} \times \vec{H}^{*}\right)
$$

is obtained

$$
\bar{S}=\frac{\omega \epsilon_{2} k_{2}^{3} C_{0}^{2} a^{2}}{32 D^{4}} F\left(\theta_{a}\right)
$$


where $F\left(\theta_{a}\right)=f^{2}\left(\theta_{a}\right)+f_{0}^{2}-2 f_{0} f\left(\theta_{a}\right) \cos \left[2 k_{2} a\left(1-\cos \theta_{a}\right)\right]$ and $\bar{S}$ is the average steady state power returned for a fixed beamwidth $2 \theta_{2 a}$ of illumination, corresponding to an illuminated area of the spherical surface subtended by the polar angle $\theta_{a}$ (measured at the origin). The angles $\theta_{a}$ and $\theta_{2 a}$ are shown in figure 5 .

Now it is desired to evaluate $C_{0}^{2}$ in terms of normal radar quantities in order to obtain the power received $P_{r}$ by the radar. If $P_{T}$ is the peak power radiated by the antenna, $G_{T}$ is the gain of the transmitting antenna over an isotropic antenna, and $G_{S D}$ is the gain of a short dipole over an isotropic antenna $(=3 / 2)$, then the power which must be radiated by the short dipole $\left(W_{S D}\right)$ to yield the same power density in the main lobe is given by

$$
W_{S D}=\frac{P_{T} G_{T}}{G_{S D}}=\frac{2 P_{T} G_{T}}{3}
$$

Then [Stratton, 1941]

$$
C_{0}^{2}=\frac{3 W_{S D}}{4 \pi \omega^{4} \epsilon_{2}^{2} \mu_{2} \sqrt{\mu_{2} \epsilon_{2}}}
$$

The received power, $P_{r}$, is given by

$$
P_{r}=\frac{\bar{S} G_{R} \lambda^{2}}{4 \pi}
$$

where $G_{R}$ is the receiving antenna gain over an isotropic antenna and $\lambda$ is the wavelength at frequency $\varpi$.

Using (32), (33), and (34), (35) becomes

$$
P_{r}\left(\theta_{a}\right)=\frac{P_{T} G_{T} G_{R} \lambda^{2}\left(\pi a^{2}\right)}{(4 \pi)^{3} D^{4}} \frac{F\left(\theta_{a}\right)}{4} .
$$

It can be observed that the third term of $F\left(\theta_{a}\right)$ as given in (32) is extremely sensitive to variations of $\theta_{a}$. This is because of the large value of $k_{2} a$ for a body of the size of the moon at microwave frequencies. Figure 6 shows $F\left(\theta_{a}\right)$ plotted versus $\theta_{a}$ for three distinct situations:

$$
\cos \left[2 k_{2} a\left(1-\cos \theta_{a}\right)\right]=1,0,-1 .
$$

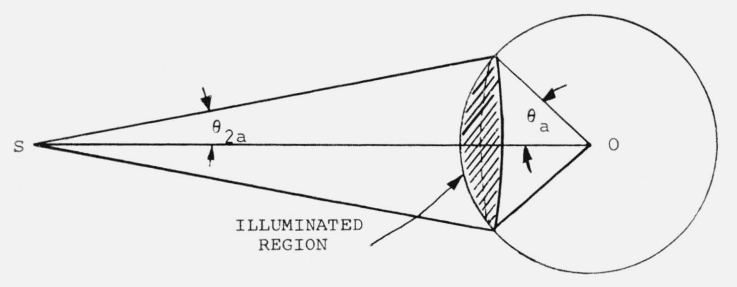

Figure 5. Beamwidth-limited illumination.

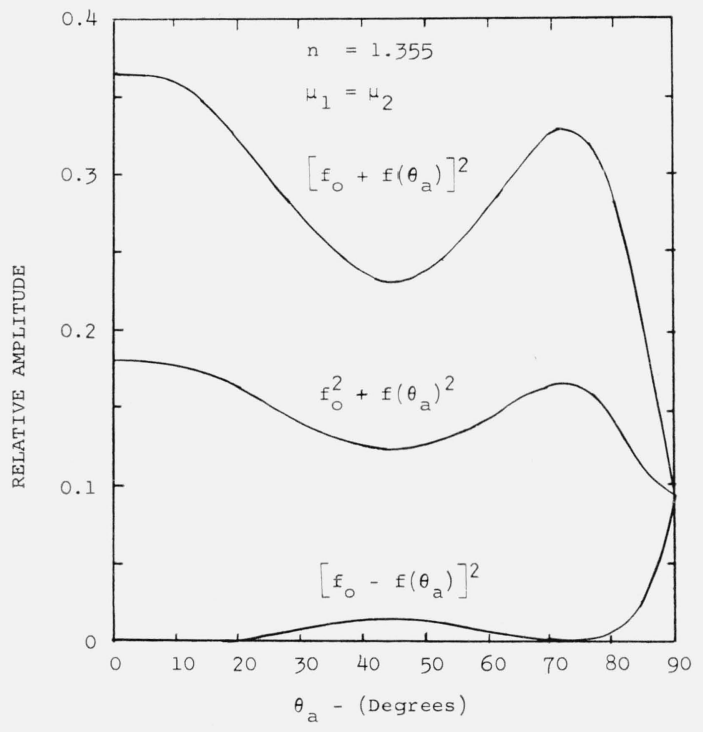

Figure 6. Relative amplitude of the envelope of $\overline{\mathrm{S}}$ versus $\theta_{\mathrm{a}}$. 
Noting that $F\left(\theta_{a}\right)$ converges to only one value for $\theta_{a}=90^{\circ}$, (36) becomes

$$
P_{r}\left(90^{\circ}\right)=\frac{P_{T} G_{T} G_{R} \lambda^{2}}{(4 \pi)^{3} D^{4}}\left(\rho^{2} \pi a^{2}\right)
$$

The last term in (38) is just the radar scattering cross section of a sphere, that is the power reflection coefficient, $\rho^{2}$, times the geometrical cross section, $\pi a^{2}$. The first part of (38) is just the standard two way radar range equation [Kerr, 1951]. Therefore, the results that are obtained in this paper using the concept of differential reflectivity are identical with those obtained under the same conditions using the Mie solution. However, using the method presented in this paper one can obtain the steady state power returned for any specific antenna pattern including partial illumination of the moon. This cannot be done by any other known method.

\section{An Application to Experimental Data}

Since it is often possible to obtain information about the transient solution of a problem from its steady state solution, an attempt is made here to predict the possible transient solution of the problem under consideration without performing the integration indicated in (3). Letting $\theta_{a}$ be considered as a function of time it is seen that $f_{0}$ in $F\left(\theta_{a}\right)$ is independent of time and will be accepted by the receiver; $f\left(\theta_{a}\right)$, is a slowly varying function of time and would, no doubt, show up in the receiver output. The last term, being a high frequency term (of the order of the transmitted frequency), would not be passed through the receiver because of its band pass characteristics. The rise time of the first two terms, however, would be too high for an ordinary radar receiver, and the initial portion of the return signal would be limited by the step response of the receiver and/or the transmitted waveform.

In addition, in the practical case of return from the moon, roughness over the first few Fresnel zones would cause a considerable modification in the received initial slope giving, possibly, an ensemble average slope of the received pulses less than that of the receiver step response. In any case the maximum of the average returned power in an experiment should be indicative of average electromagnetic properties of the moon's surface for the assumption that the surface roughness is negligible. If the roughness were not negligible, the maximum average value would be less than that for the case involving no roughness. Correspondingly, the average electromagnetic properties obtained by matching the maximum ensemble average of actual returns with the maximum indicated by this theory (for a smooth moon), would yield the minimum average values for the actual moon.

The received power will now be calculated and applied to the data obtained from a pulse radar lunar reflections experiment by Mathis [1963]. By the previous discussion only the $D C$ and slowly varying terms of $F\left(\theta_{a}\right)$ will be used in the computation of $P_{r}$. The radar parameters of Mathis' experiment are listed in table 1. The maximum value of $F\left(\theta_{a}\right)$ is

Then (36) becomes

$$
\left[f_{0}^{2}+f^{2}\left(\theta_{a}\right)\right]_{\max }=8 \rho^{2} .
$$

$$
P_{r_{\max }}=\frac{G_{T} G_{R} P_{T} \lambda^{2}}{(4 \pi)^{3} D^{4}}\left(2 \rho^{2} \pi a^{2}\right)
$$

and on letting

$$
\begin{aligned}
P_{T} & =2.5 \times 10^{6} \mathrm{~W} \\
D & =2.34 \times 10^{5} \text { miles } \\
a & =1.08 \times 10^{3} \text { miles } \\
G_{R} & =G_{T}=37.5 \mathrm{~dB} \\
P_{r_{\max }}(\mathrm{dBm}) & =-77.3+20 \log _{10} \rho .
\end{aligned}
$$


Now using Mathis' [1963] maximum value for $P_{r_{\max }}$ of $-96.8 \mathrm{dBm}$, one obtains $|\rho|=0.106$ and upon letting $\mu_{1}=\mu_{2}$

$$
n=\frac{1+|\rho|}{1-|\rho|}=1.24=\sqrt{\frac{\epsilon_{1}}{\epsilon_{2}}}
$$

This yields a minimum average value of $\epsilon$ for the material composing the moon's surface. Thus $\epsilon_{\mathrm{avg}}=1.53 \epsilon_{0}$, a value which is consistent with those obtained by other investigators.

TABLE 1. Trinidad test site radar parameters

[Mathis, 1963]

\begin{tabular}{|c|c|}
\hline Antenna & $\begin{array}{l}84 \mathrm{ft} \text { parabolic dish on } \mathrm{Az}-\mathrm{E} 1 \text { mount } \\
37.5 \mathrm{~dB} \text { gain } \\
2.25^{\circ} \text { beamwidth } \\
\text { Transmit one polarization } \\
\text { Receive transmitted and orthogonal polarization }\end{array}$ \\
\hline Transmitter & $\begin{array}{l}\text { Continental electronics } \mathrm{AN} / \mathrm{FPT}-5 \\
425.0 \mathrm{Mc} / \mathrm{s} \\
2000 \mu \mathrm{sec} \text { pulse } \\
30 \text { or } 30.2 \text { pulses/sec } \\
2.5 \mathrm{MW} \text { peak power (nominal) } \\
\text { Rectangular pulse }\end{array}$ \\
\hline Receivers & $\begin{array}{l}\text { Noise figure } 5.5 \mathrm{~dB} \text { maximum } \\
\text { Frequency stability }-1 \text { part in } 10 \text { in } 33 \mathrm{msec} \\
\text { Gain stability }-0.25 \mathrm{~dB} \text { in } 4 \mathrm{hr} \\
\text { Dynamic range } 40 \mathrm{~dB} \text { (linear) } \\
\text { Bandwidth } 4.7 \mathrm{kc} / \mathrm{s}\end{array}$ \\
\hline
\end{tabular}

\section{Conclusions}

This paper has presented an exact solution to beam-limited reflections of a conically bounded spherical wave from a semi-infinite plane through the use of the differential reflectivity. In general, the use of differential reflectivity provides a method for decomposing the radiation from an arbitrary source into an infinite set of plane waves at each point of the illuminated area so that, in order to determine the reflected fields, only the reflection coefficients for a plane wave incident on the body need be known or approximated. It has been qualitatively shown that the approximate reflection coefficients for a convex body with either a large radius of curvature or consisting of a lossy medium are the Fresnel coefficients.

The theory has been applied to the case in which a beam-limited spherical wave is incident on a smooth sphere. The steady state backscattered power returned from the sphere is obtained. The time average steady state power returned displays very rapid fluctuations with the increase in the number of Fresnel zones illuminated. Upon matching this expression for average power with the appropriate experimental data as obtained from the moon, the minimum value for the average dielectric constant is found to be 1.53 .

This method, using the differential reflectivity, proves to be a useful tool which can also be applied directly to the case of acoustic scattering. With the use of high-speed digital computers, the restrictions imposed herein merely to facilitate computation can be easily relaxed. The method may also be easily modified so as to incorporate statistical variations in any electrical or geometrical parameters.

\section{References}

Beckmann, P., and A. Spizzichino (1963), The Scattering of Electromagnetic Waves from Rough Surfaces, ch. 20 (Pergamon Press).

Brekhovskikh, L. M. (1960), Waves in Layered Media, ch. 4 (Academic Press).

Kerr, D. E. (1951), Propagation of Short Radio Waves, ch. 6 (McGraw-Hill Book. Co., Inc., New York, N.Y.). Mathis, R. C. (1963), A lunar echo study at $425 \mathrm{Mc} / \mathrm{s}$, Ph. D. dissertation, The University of Texas (AD415812).

Stratton, J. A. (1941), Electromagnetic Theory, ch. 8 and 9 (McGraw-Hill Book Co., Inc., New York, N.Y.). 